\title{
Hepatic Abscess as Early Presentation of Perforated Diverticulitis
}

\author{
Gauthier Stepman $^{\mathrm{a}, \mathrm{b}}\left(\mathbb{D}\right.$, Jinal K. Patel ${ }^{\mathrm{a}}$, Jordan Young ${ }^{\mathrm{a}}$, Johnathan Frunzi ${ }^{\mathrm{a}}$
}

\begin{abstract}
A hepatic abscess is a rare condition which can have multiple etiologies, including biliary disease, intra-abdominal collections (appendicitis, diverticulitis, and inflammatory bowel disease), abdominal surgery, liver transplantation, and liver trauma. The diagnosis is primarily based on imaging findings of ultrasound and computed tomography scan. Management includes antibiotics and percutaneous drainage or surgical drainage of the abscess. Here, we present a case of a 43-year-old Hispanic female who initially presented with left lower quadrant and right upper quadrant abdominal pain for 1 week. She was found to have a hepatic abscess and pelvic abscess likely secondary to perforated diverticulum.
\end{abstract}

Keywords: Hepatic abscess; Perforated diverticulitis; CT; Complication

\section{Introduction}

Hepatic abscesses can occur by microbial contamination of the liver parenchyma. The infection is most commonly bacterial [1]. Hepatic abscesses have multiple causes, including biliary disease, intra-abdominal collections (appendicitis, diverticulitis, and inflammatory bowel disease), abdominal surgery, liver transplantation, and liver trauma [1-4]. Hepatic abscesses are often complicated by septic shock [2].

Diverticulitis is an inflammatory process of diverticula of the colonic wall. When diverticulitis is associated with an abscess, fistula, perforation, or obstruction, it is considered to be complicated [3]. Diverticulitis is thought to cause hepatic abscesses by portal venous route when rupture is present [4].

Diagnosis of hepatic abscesses is via imaging modality, including ultrasound, computed tomography (CT) and mag-

Manuscript submitted August 2, 2021, accepted September 21, 2021

Published online November 5, 2021

aDepartment of Internal Medicine, HCA Healthcare/USF Morsani College of Medicine GME: Medical Center of Trinity, Trinity, FL, USA

${ }^{b}$ Corresponding Author: Gauthier Stepman, Department of Internal Medicine, HCA Healthcare/USF Morsani College of Medicine GME: Medical Center of Trinity, Trinity, FL, USA. Email: Gauthier.Stepman@hcahealthcare.com

doi: https://doi.org/10.14740/jmc3762 netic resonance imaging (MRI) [1]. Confirmation is obtained by needle aspiration with identification of the causative organism [1]. Treatment modalities include antibiotics and drainage of the abscess (ultrasound-guided or surgical).

\section{Case Report}

\section{Investigations}

Our patient is a 43-year-old Hispanic female with no significant medical history. She presented to the hospital with right upper quadrant (RUQ) abdominal pain for 1 week. She also complained of fever, nausea, vomiting, and diarrhea for approximately 1 week. Upon presentation, she was found to be hypotensive and tachycardic, not responding to fluid boluses. Her initial vitals were as follows: temperature of 38.0 ${ }^{\circ} \mathrm{C}$, pulse of 138 beats per minute, respiratory rate of 18 per minute, blood pressure of $90 / 54 \mathrm{~mm} \mathrm{Hg}$, and pulse oximetry of $99 \%$ on room air. Physical examination results were pertinent for dry mucosal membranes and diffuse abdominal tenderness. She was transferred to the intensive care unit with septic shock.

\section{Diagnosis}

Her initial lab values revealed a white blood cell count of $5,620 / \mu \mathrm{L}$, hemoglobin of $11.7 \mathrm{~g} / \mathrm{dL}$, sodium level of 130 $\mathrm{mmol} / \mathrm{L}$, potassium of $3.3 \mathrm{mmol} / \mathrm{L}$, creatinine of $3.3 \mathrm{mg} / \mathrm{dL}$, glomerular filtration rate of $15.26 \mathrm{~mL} / \mathrm{min}$, total bilirubin of $1.5 \mathrm{mg} / \mathrm{dL}$, aspartate aminotransferase of $246 \mathrm{U} / \mathrm{L}$, alanine aminotransferase of $185 \mathrm{U} / \mathrm{L}$, alkaline phosphatase of 131 $\mathrm{U} / \mathrm{L}$, lipase of $35 \mathrm{U} / \mathrm{L}$ and a lactic acid of $7.2 \mathrm{mmol} / \mathrm{L}$. She was also found to be coronavirus disease 2019 (COVID-19) positive by polymerase chain reaction testing. Since she did not require supplemental oxygen, no specific treatment for COVID-19 was given.

A CT scan of the abdomen was obtained in the emergency department and revealed a poorly defined hypodense cystic structure in the right liver lobe (Fig. 1), but no other acute intra-abdominal pathology. A follow-up ultrasound was performed and revealed a $9.9-\mathrm{cm}$ mass in the right lobe of the liver (Fig. 2). A follow-up MRI scan of the abdomen revealed a heavily septated $13-\mathrm{cm}$ hepatic abscess in the posterior right hepatic lobe (Fig. 3). 


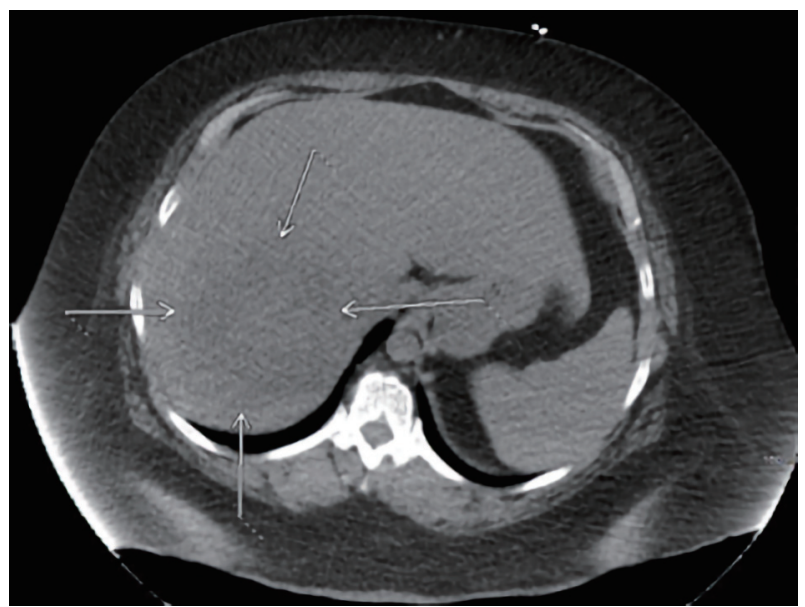

Figure 1. Computed tomography (CT) scan of the abdomen showing the liver abscess (arrows).

\section{Treatment}

In the emergency department she was given multiple fluid boluses and was started on intravenous (IV) vancomycin and piperacillin/tazobactam.

Percutaneous drainage was performed and yielded 1,110 $\mathrm{mL}$ of purulent fluid over the course of 8 days. The CT scan for the needle placement also revealed a small pneumoperitoneum. Cultures revealed Streptococcus viridans as the causative organism. The antibiotics were switched to ampicillin/sulbactam. The patient's RUQ pain improved significantly during her hospital course. On discharge, the antibiotic regimen for the hepatic abscess was changed to amoxicillin to allow for an oral regimen based on the sensitivities.

\section{Follow-up and outcomes}

On hospital day 8 , the patient complained of left lower quad-

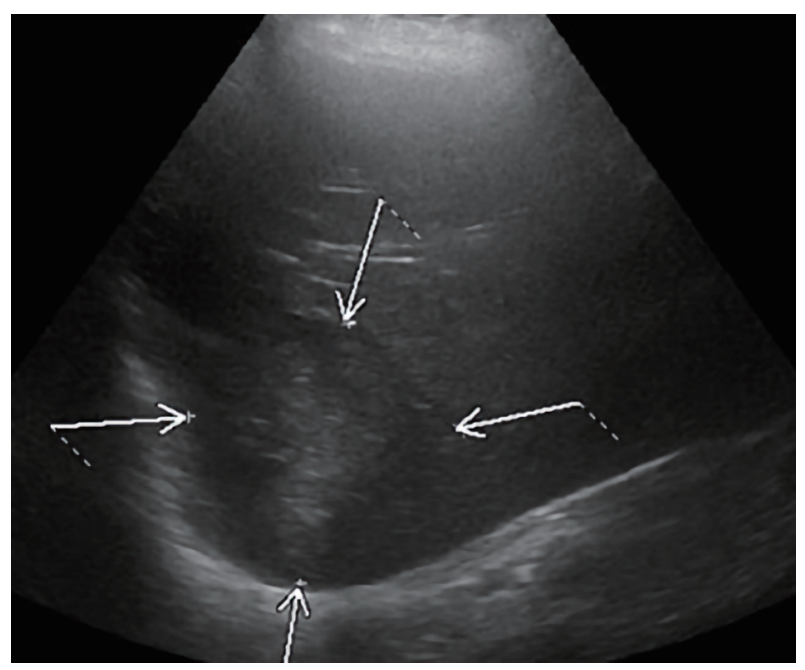

Figure 2. Ultrasound of the liver showing the liver abscess (arrows).

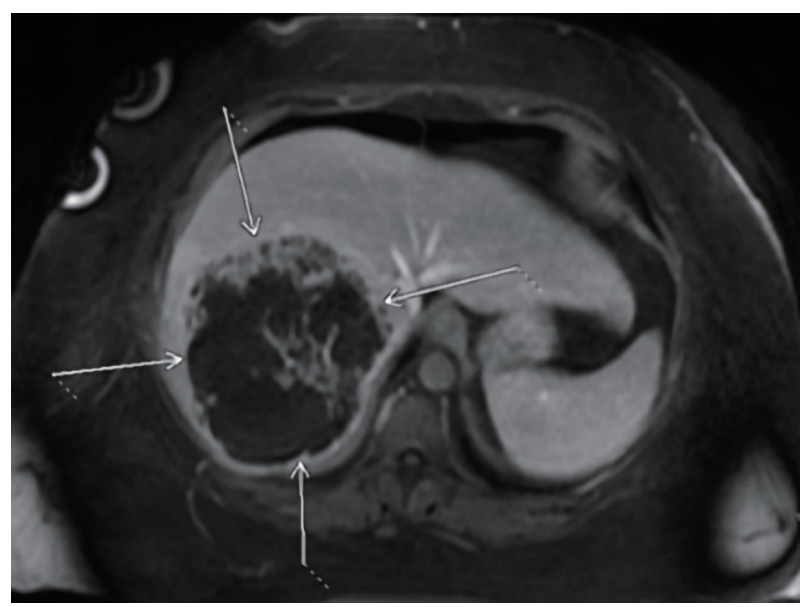

Figure 3. Magnetic resonance imaging (MRI) of the abdomen showing a heavily septated $13-\mathrm{cm}$ hepatic abscess in the posterior right hepatic lobe (arrows).

rant (LLQ) abdominal pain. A repeat $\mathrm{CT}$ of the abdomen revealed a decreased size of the liver lesion, a pelvic abscess measuring $13.6 \times 5 \mathrm{~cm}$ (Fig. 4), and unchanged pneumoperitoneum, likely secondary to a perforated sigmoid diverticulum. She again underwent CT-guided percutaneous drainage. Cultures from the pelvic abscess revealed mixed gram-positive and gram-negative flora and Candida albicans. The patient was subsequently discharged with both drains in place and a 20-day course of amoxicillin and fluconazole.

The patient returned to the hospital 10 days later for drain removal and reported improvement of her LLQ pain.

\section{Discussion}

Hepatic abscesses are relatively rare, with incidence being as low as 20 per 100,000 admissions [1]. Despite the rarity, it is associated with high mortality rates, up to $15 \%[1,2]$.

In a case series published by Kubovy et al, diverticulitis

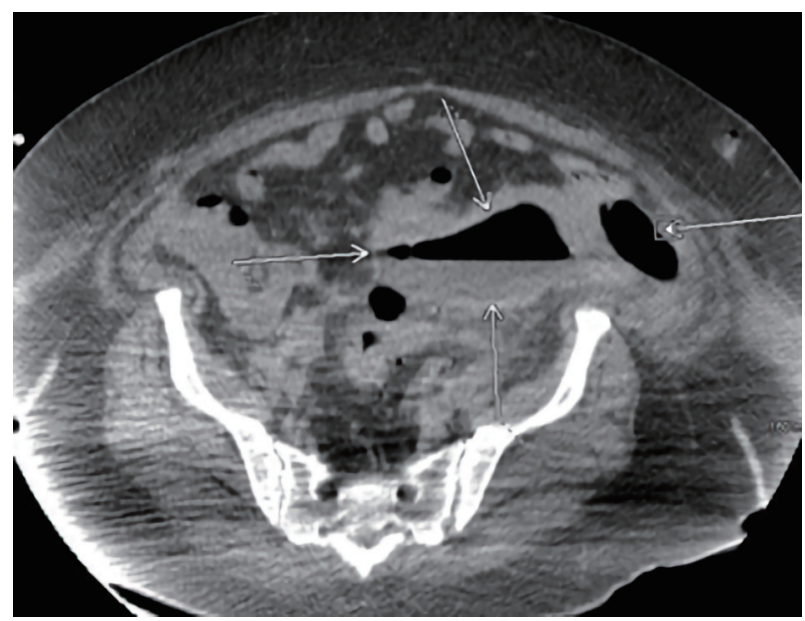

Figure 4. Repeat computed tomography (CT) scan of the abdomen. 
was found to be the cause in $12 \%$ [2]. Out of the 25 cases reported only three patients had underlying diverticulosis/diverticulitis [2]. However, it is not clear if acute diverticulitis was present at the time of the hepatic abscess [2]. This case series noted diabetes mellitus, hypertension, and atrial fibrillation to be the most common comorbidities [2]. Our patient differed from the published case series in several ways. Our patient did not have any preexisting conditions, including a history of diverticulitis, she was a Hispanic female, and was much younger than the average patient in the published case series [2].

Our patient's case is unique as the liver abscess was identified several days prior to the perforated diverticulitis. To our knowledge, this is only the second reported case of a hepatic abscess preceding the diagnosis of sigmoid diverticulitis [5]. Our patient and the patient reported by Murarka et al both had interval development of pelvic abscesses secondary to diverticulitis, despite the lack of personal history of diverticulitis [5]. However, several differences were noted between our case and the case of Murarka et al [5]. They reported a 71-yearold Caucasian male with Streptococcus anginosus bacteremia, multiple liver abscesses, and portal vein thrombosis [5]. Our patient was a 43-year-old female without bacteremia and a single hepatic abscess. Portal vein thrombosis is a frequent complication related to hepatic abscesses $[1,5,6]$. Our patient did not have any venous thromboembolism. It is unknown if our patient underwent a colonoscopy as an outpatient following her episode of diverticulitis.

The presentation of a hepatic abscess can resemble acute cholangitis, with symptoms such as fever, RUQ pain, jaundice, and weight loss [1, 4]. Bacteremia can also be present [5]. However, the presentation is diverse and often includes symptoms from the underlying disease [1]. Our patient did have fever and RUQ pain, but no jaundice. She also did not have bacteremia. She also had LLQ pain associated with her sigmoid diverticulitis.

The causative organism in bacterial hepatic abscesses is often identified as Streptococcus intermedius (S. intermedius) $[2,4,5]$. The hepatic abscess in our patient was also found to have been caused by $S$. intermedius. The sigmoid abscess was drained with CT-guided needle aspiration.

The culture did not specifically reveal $S$. intermedius, but rather mixed gram-positive and gram-negative flora as well as Candida albicans. The reason for this discrepancy is unknown. She did receive antibiotics for several days prior to aspiration of the pelvic abscess, which could have altered the microbiological profile of the abscess.

Diagnosis of a hepatic abscess is based on imaging, with over $90 \%$ of cases being identified with sonography and CT scan [1]. Our patient underwent both sonography and CT imaging, but also underwent MRI imaging to further evaluate the hepatic abscess.

Initial management of a single-cavity hepatic abscess consists of percutaneous drainage and antibiotics [1-6]. If multiple hepatic abscesses are present or if the hepatic abscess is loculated, surgical drainage might be necessary [2, 4]. Our patient fared well with percutaneous drain placement, 10 days of IV antibiotics and a 20-day course of oral amoxicillin. A prolonged course of both IV and oral antibiotic treatment is often needed [2-6].

\section{Conclusions}

Hepatic abscesses are a rare but potentially fatal complication of perforated diverticulitis. Associated with significant morbidity, presentation may resemble acute cholangitis, but can be diverse and nonspecific. In the presence of LLQ pain, diverticulitis as cause must be presumed, even in the absence of initial imaging findings to support diverticulitis. Physicians should have a low threshold to repeat abdominal imaging if suspicion for diverticulitis is high.

\section{Acknowledgments}

None to declare.

\section{Financial Disclosure}

This research was supported in part by HCA Healthcare and/ or an HCA Healthcare affiliated entity. The views expressed in this publication represent those of the author(s) and do not necessarily represent the official views of HCA Healthcare or any of its affiliated entities.

\section{Conflict of Interest}

The authors confirm that there is no conflict of interest.

\section{Informed Consent}

Informed consent was obtained from the patient.

\section{Author Contributions}

GS: introduction and case presentation. JKP: discussion and abstract. JY and JF: essential feedback and edits.

\section{Data Availability}

The authors declare that data supporting the findings of this study are available within the article.

\section{Abbreviations}

CT: computed tomography; MRI: magnetic resonance imaging; RUQ: right upper quadrant

\section{References}

1. Lardiere-Deguelte S, Ragot E, Amroun K, Piardi T, Dok- 
mak S, Bruno O, Appere F, et al. Hepatic abscess: Diagnosis and management. J Visc Surg. 2015;152(4):231243.

2. Kubovy J, Karim S, Ding S. Pyogenic liver abscess: incidence, causality, management and clinical outcomes in a New Zealand cohort. N Z Med J. 2019;132(1492):30-35.

3. Navarrete D, Patil S, Dandachi D. Acute Streptococcus constellatus Pyogenic Liver Abscess Due to an Atypical Presentation of Sigmoid Diverticulitis Complicated by Pericolonic Abscess. Cureus. 2020;12(10):e10940.
4. Muscat EPJ. Pyogenic liver abscess secondary to sigmoid diverticulitis: an unusual presentation. Case Rep Gastroenterol. 2020;14(1):165-171.

5. Murarka S, Pranav F, Dandavate V. Pyogenic liver abscess secondary to disseminated streptococcus anginosus from sigmoid diverticulitis. J Glob Infect Dis. 2011;3(1):79-81.

6. Al Hajjar N, Crisan D, Grigorescu M, Boruah P. Acute diverticulitis - an unusual cause of liver abcesses in a young man: a case report. Chirurgia (Bucur). 2012;107(2):267272. 